\title{
Anosognosia pós Acidente Vascular Cerebral: percepção do paciente e familiar
}

\author{
Anosognosia post stroke: \\ perception of the patient and family
}

\section{Anosognosia después de un accidente cerebrovascular: percepción del paciente y la familia}

\author{
Gabriela Marques Dias ${ }^{1}$, Morgana Christmann²
}

\begin{abstract}
1.Fisioterapeuta, Especialização pela Residência Multiprofissional em Atenção Clínica Especializada com Ênfase em Infectologia e Neurologia, Universidade Franciscana, Santa Maria-RS, Brasil. Orcid: http://orcid.org/0000-0003-0045-3731

2.Fisioterapeuta, Professora Doutora da Residência Multiprofissional em Atenção Clínica Especializada com ênfase em Infectologia e Neurologia, Universidade Franciscana, Santa Maria-RS, Brasil. Orcid: http://orcid.org/0000-0002-5773-8933
\end{abstract}

\begin{abstract}
Resumo
Introdução. A anosognosia é a alteração da percepção da existência das dificuldades. Objetivo. Avaliar a anosognosia pós Acidente Vascular Cerebral (AVC) nas fases aguda e subaguda (até seis meses após a lesão). Método. A amostra foi constituída por pacientes atendidos em um Hospital ou na Atenção Primária à Saúde, e seus familiares. Foi um estudo quantitativo, de desenho transversal, a coleta de dados ocorreu de agosto a novembro de 2020. Foram aplicados aos pacientes e familiares os questionários Dados Sociodemográficos e The Patient Competency Rating Scale (PCRS), ambos em duas versões. Resultados. A amostra apresentou 10 pacientes e familiares, os pacientes tinham média de idade $55,4 \pm 15$. $\mathrm{Na}$ observação da discrepância da pontuação PCRS entre pacientes e familiares, dois (20\%) dos pacientes não apresentam anosognosia e três (30\%) apresentam pouca discrepância, considerou-se que não apresentam anosognosia. Já cinco (50\%) dos pacientes tiveram discrepância na PCRS com os familiares e considerou-se que apresentam anosognosia. Não é possível afirmar estatisticamente, sugerimos que pontuações discrepantes maior que três sugerem anosognosia. Conclusão. No presente estudo, 20\% dos pacientes com AVC não possuem anosognosia por não apresentarem diferença na PCRS entre paciente e familiar, assim como os $30 \%$ pela mínima diferença nos resultados. Entre os fatores relacionados à autopercepção positiva do seu estado de saúde se destacou como elemento considerável para a presença de anosognosia. Em nosso estudo, a anosognosia pode estar relacionada a idade mais avançada, uso de antidepressivo ou ansiolítico, hemiparesia à direita, maior dependência para locomoção e influência do tabagismo e etilismo.
\end{abstract}

Unitermos. Acidente Vascular Cerebral; Anosognosia; Hemiplegia

\footnotetext{
Abstract

Introduction. Anosognosia is the change in the perception of the existence of difficulties. Objective. To assess anosognosia after stroke in the acute and subacute phases (up to six months after the injury). Method. The sample consisted of patients seen in a Hospital or in Primary Health Care, and their relatives. It was a quantitative, cross-sectional study, data collection took place from August to November 2020. The Sociodemographic Data and The Patient Competency Rating Scale (PCRS) questionnaires, both in two versions, were applied to patients and family members. Results. The sample had 10 patients and family members, the patients had na average age of $55.4 \pm 15$. In observing the discrepancy in the PCRS score between patients and family members, two (20\%) of the patients do not have anosognosia, and three $(30 \%)$ have little discrepancy, it was considered that they do not have anosognosia. Now, five $(50 \%)$ of the patients had discrepancies in the PCRS with their relatives and it was considered that they have anosognosia. We do not state statistically, we suggest that
} 
discrepant scores greater than three suggest anosognosia. Conclusion. No study was present, $20 \%$ of two patients with stroke did not present anosognosia for not presenting a difference in the PCRS between patient and family, as well as $30 \%$ with minimal difference in the results. Among the factors related to the positive self-perception of their health status, it stands out as a considerable element for the presence of anosognosia. In our study, anosognosia may be related to more advanced disease, use of antidepressants or anxiolytics, direct hemiparesis, greater dependence for locomotion and the influence of smoking and alcoholism.

Keywords. Stroke; Anosognosia; Hemiplegia

\begin{abstract}
Resumen
Introducción. La anosognosia es un cambio en la percepción de la existencia de dificultades. Objetivo. Evaluar la anosognosia tras un ictus en las fases aguda y subaguda (hasta seis meses después de la lesión). Método. La muestra estuvo constituida por pacientes atendidos en un Hospital o en Atención Primaria de Salud y sus familiares. Fue un estudio cuantitativo, transversal, la recolección de datos se llevó a cabo de agosto a noviembre de 2020. Se aplicaron los cuestionarios Datos Sociodemográficos y Escala de Calificación de Competencia del Paciente (PCRS), ambos en dos versiones, a pacientes y familiares. Resultados. La muestra estuvo compuesta por 10 pacientes y familiares, los pacientes tenían una edad media

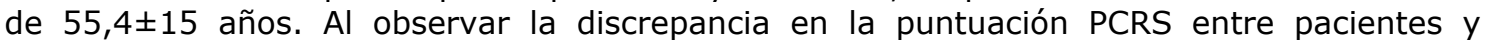
familiares, dos (20\%) de los pacientes no tienen anosognosia, y tres (30\%) tienen poca discrepancia, se consideró que no tienen anosognosia. Ahora, cinco (50\%) de los pacientes tenían discrepancias en la PCRS con sus familiares y se consideró que tienen anosognosia. No declaramos estadísticamente, sugerimos que las puntuaciones discrepantes superiores a tres sugieren anosognosia. Conclusiones. En el presente estudio, el 20\% de los pacientes con ictus no tienen anosognosia porque no tienen diferencia en la PCRS entre paciente y familiar, así como el $30 \%$ por la más mínima diferencia en los resultados. Entre los factores relacionados con la autopercepción positiva de su estado de salud, se destacó como un elemento considerable para la presencia de anosognosia. En nuestro estudio, la anosognosia puede estar relacionada con la edad avanzada, el uso de antidepresivos o ansiolíticos, la hemiparesia derecha, una mayor dependencia para la locomoción y la influencia del tabaquismo y el consumo de alcohol.
\end{abstract}

Palabras clave. Accidente cerebrovascular; Anosognosia; Hemiplejía

Trabalho realizado no Hospital Casa de Saúde; Laboratório de Ensino Prático (LEP) em Fisioterapia da Universidade Franciscana (UFN), Santa Maria-RS, Brasil.

\title{
INTRODUÇÃO
}

\section{O Acidente Vascular Cerebral (AVC) é um dos} problemas de saúde pública mais relevantes no mundo, sendo a segunda causa mais frequente de óbito ${ }^{1}$. O AVC é classificado em fases de acordo com o tempo após a lesão: agudo que compreende o primeiro mês, subagudo, entre um mês e seis meses, e crônico a partir de seis meses ${ }^{2}$. Essa distinção varia entre os autores ${ }^{3}$. 
Após o AVC os indivíduos podem apresentar comprometimentos neurológicos importantes como, déficit cognitivo e mental, afasia, alteração na propriocepção, alterações motoras e sensitivas, déficits no equilíbrio e na marcha, redução da força muscular, entre outros ${ }^{4}$.

O termo anosognosia é descrito como a alteração de indivíduos quanto a percepção da existência ou extensão de suas dificuldades ${ }^{5}$. A anosognosia é uma alteração no processamento visuoespacial que resulta na heminegligência do lado contralateral à lesão cerebral, juntamente com a inexistência de consciência e auto-percepção desse déficit ${ }^{6}$.

Após um AVC como complicação neuropsicológica podese destacar a anosognosia, especialmente na fase aguda?. No que tange a epidemiologia, observa-se com mais frequência após danos no hemisfério cerebral direito, repercutindo no hemicorpo esquerdo 8 .

$\mathrm{Na}$ perspectiva da literatura sobre o tema, poucos estudos buscam aprofundar a temática da anosognosia em específico ${ }^{9}$, mencionando como um elemento parte das sequelas do $A V C$, sem especificidades ${ }^{10-12}$, fato que justifica a importância deste estudo.

A anosognosia pode ocorrer após lesões cerebrais, podendo causar prejuízos na vida dos indivíduos, como o não engajamento na reabilitação e reduzida melhora na reabilitação. $O$ estudo, portanto, tem como objetivo fornecer informações quanto à existência e fatores relacionados à anosognosia pós AVC nas fases aguda e subaguda (até seis meses de lesão). 


\section{MÉTODO}

\section{Amostra}

Este estudo quantitativo, teve caráter descritivo exploratório e de desenho transversal, com pacientes com diagnóstico médico de AVC nas fases aguda e subaguda, ou seja, até seis meses após a lesão², uma vez que a literatura sugere que este é o período com maior incidência anosognosia após $\mathrm{AVC}^{13}$ e seus respectivos familiares sendo a amostra por conveniência. A coleta de dados foi realizada em um hospital municipal do interior do estado do Rio Grande do Sul, em uma Unidade Básica de Saúde (UBS) e uma Estratégia de Saúde da Família (ESF), vinculados às práticas do Curso de Fisioterapia da Universidade Franciscana (UFN). A coleta de dados foi realizada durante os meses de agosto a novembro de 2020.

Foram incluídos na pesquisa os pacientes e familiares de ambos os sexos e diversas idades, que aceitaram participar do estudo no período da coleta de dados e que possuíam linguagem verbal preservada. Além disso, foi aplicado o Mini Exame do Estado Mental (MEEM), como critério de inclusão dos pacientes e familiares, tendo como limiar base a escolaridade. O MEEM utiliza como parâmetro o escore 29 para pessoas com pelo menos nove anos de escolaridade, 26 para aqueles com cinco a oito anos de escolaridade, e 22 para os com zero a quatro anos de escolaridade ${ }^{14}$. O MEEM foi utilizado como critério de inclusão do estudo por avaliar o nível cognitivo dos participantes, e 
consequentemente proporcionar fidedignidade nas respostas dos pacientes e familiares nas escalas aplicadas.

Foram excluídos da pesquisa os pacientes e familiares que possuíam outras patologias neurológicas associadas (Alzheimer, Parkinson, traumatismo cranioencefálico, ou qualquer outra condição neurológica que interferia na compreensão para responder os questionários), não tivessem acompanhantes, não aceitaram participar do estudo e não possuam linguagem verbal.

A pesquisa teve início após aprovação do Comitê de Ética em Pesquisa (CEP) da Universidade Franciscana (UFN) sob o número do parecer de aprovação $n^{\circ}$ 4.220.818. A captação dos participantes do estudo foi feita por convite com uma linguagem simples, a cada paciente e familiar que se encaixaram nos critérios de inclusão. Previamente foram explicados os objetivos, a metodologia, os riscos e benefícios da pesquisa contidos no Termo de Consentimento Livre e Esclarecido (TCLE). Para após o aceite da participação ser assinado o TCLE pelos indivíduos.

\section{Procedimento}

$\mathrm{Na}$ coleta de dados dos pacientes internados no Hospital, observou-se o mural do Posto de Enfermagem, em que constava os pacientes internados e o diagnóstico médico, e também analisou o prontuário eletrônico dos pacientes, na busca pelo diagnóstico clínico de AVC nas fases aguda e subaguda. Já, para acessar os pacientes vinculados às equipes da ESF e/ou UBS, foi realizado contato com as 
mesmas e a coleta de dados foi agendada no Laboratório de Ensino Prático em Fisioterapia (Lep) na UFN, no domicílio dos participantes ou na modalidade on-line, definido em comum acordo.

$\mathrm{Na}$ coleta de dados dos pacientes foi realizado o preenchimento do questionário com os dados Sociodemográficos e o questionário The Competency Rating Scale (PCRS) para Pacientes na versão traduzida e adaptada para o português ${ }^{15}$. Para a coleta de dados dos familiares foi preenchido o questionário com os Dados Sociodemográficos e PCRS na versão em português para familiares.

O questionário de Dados Sociodemográficos, desenvolvidos pelos autores compreendiam 17 perguntas fechadas sobre dados de identificação (idade, sexo, raça, estado civil e anos de estudo), comorbidades, uso de medicamentos, o AVC (tempo de internação hospitalar, tempo do AVC, tipo do AVC, AVC prévio, região cerebral acometida, localização da hemiparesia), hábitos de vida (tabagismo, etilismo), locomoção e pontuação do MEEM. No levantamento dos Dados Sociodemográficos dos Familiares desenvolvidos pelos autores, compreendiam 8 perguntas fechadas sobre dados de identificação (idade, sexo, raça, estado civil, anos de estudo), possui trabalho, parou de trabalhar para cuidar do paciente e pontuação do MEEM.

A PCRS possui como objetivo analisar a consciência de déficits dos indivíduos acometidos por patologias neurológicas. Essa é composta por 17 itens, a pontuação varia de 17 a 85 , apresenta duas versões, sendo uma para 
o paciente, que trata sobre suas percepções em relação ao seu corpo e estado de saúde e, outra para o familiar sobre o paciente. É avaliada a discrepância entre as respostas de familiares e pacientes, em que a resposta do familiar é a referência. A classificação é realizada da seguinte maneira: 1- não consegue, 2- muito difícil, 3- faz com alguma dificuldade, 4- razoavelmente fácil e 5- faz com facilidade ou 5- não se aplica. Essa escala não possui escore de classificação, portanto foi avaliada a diferença entre a soma total das respostas do paciente e familiar, e se o paciente se enxerga de maneira positiva ou negativa ${ }^{15}$.

\section{Análise Estatística}

Os dados foram tabulados em uma planilha do Microsoft Excel e após foi realizada a estatística descritiva das variáveis avaliadas, a fim de estruturar um perfil da amostra estudada.

\section{RESULTADOS}

Entre os pacientes internados no Hospital com diagnóstico de AVC nas fases aguda e subaguda foram excluídos 20 pacientes da coleta de dados e 19 acompanhantes/familiares. Os motivos da exclusão foram a existência de doença neurológica associada, que comprometesse a compreensão dos questionários $(n=6)$, alteração na linguagem verbal que interferisse na resposta aos questionários $(n=11)$, sem acompanhante $(n=1)$, paciente não alcançou o ponto de corte do $\operatorname{MEEM}(n=1)$, e 
acompanhante desorientada $(n=1)$. Foi considerado perda amostral aqueles que tiveram internação de curto tempo $(n=2)$, que não foi possível realizar a coleta. Entre os pacientes vinculados à UBS ou ESF com AVC nas fases aguda e subaguda foram excluídos cinco pacientes por não alcançarem o ponto de corte do MEEM.

Portanto, foram incluídos no estudo 10 pacientes e familiares, destes três estavam internados no Hospital e sete estavam vinculados à UBS ou ESF, que alcançaram o ponto de corte do MEEM, e consequentemente foi aplicada a PCRS para investigação da anosognosia.

A amostra de pacientes foi caracterizada deste modo com média de idade de $55 \pm 15$ anos, os quais seis (60\%) são do sexo masculino, cinco (50\%) são casados, cinco (50\%) tem de cinco a oito anos de estudo, 10 (100\%) tiveram AVC isquêmico, sete (70\%) não tinham AVC prévio e tiveram pontuação média no MEEM de $25 \pm 2$. Na Tabela 1 são apresentados os dados sociodemográficos dos pacientes com a porcentagem, média e desvio padrão (DP) das variáveis.

Foram avaliados 10 familiares, com média de idade de $42 \pm 11$ anos, os quais oito (80\%) são do sexo feminino, seis $(60 \%)$ são casados, seis (60\%) trabalham, apenas um (10\%) parou de trabalhar para tornar-se cuidador. Na Tabela 2 são apresentados os dados sociodemográficos dos familiares. 
Tabela 1. Caracterização sociodemográfica dos pacientes.

\begin{tabular}{|c|c|c|c|c|}
\hline Variáveis & & $\mathbf{N}$ & $\%$ & Média e DP \\
\hline Idade & & & & $55,4 \pm 15,61$ \\
\hline \multirow[t]{2}{*}{ Sexo } & Feminino & 6 & 60 & \\
\hline & Masculino & 4 & 40 & \\
\hline \multirow[t]{4}{*}{ Raça/cor } & Branca & 2 & 20 & \\
\hline & Parda & 2 & 20 & \\
\hline & Preta & 1 & 10 & \\
\hline & Outra & 5 & 50 & \\
\hline \multirow[t]{4}{*}{ Estado civil } & Solteiro & 3 & 30 & \\
\hline & Casado & 5 & 50 & \\
\hline & Separado & 1 & 10 & \\
\hline & Outro & 1 & 10 & \\
\hline \multirow{3}{*}{ Anos de estudo } & 0 a 4 & 4 & 40 & \\
\hline & 5 a 8 & 5 & 50 & \\
\hline & Mais de 9 & 1 & 10 & \\
\hline \multirow[t]{9}{*}{ Comorbidades } & HAS & 7 & 70 & \\
\hline & Fibrilação Atrial & 2 & 20 & \\
\hline & Doença Arterial Coronariana & 1 & 10 & \\
\hline & Diabetes & 2 & 20 & \\
\hline & Depressão & 1 & 10 & \\
\hline & Obesidade & 2 & 20 & \\
\hline & Arritmia Cardíaca & 1 & 10 & \\
\hline & Flutter Atrial & 1 & 10 & \\
\hline & Insuficiência Cardíaca Congestiva & 1 & 10 & \\
\hline \multirow[t]{7}{*}{ Medicamentos } & Antidiabético & 2 & 20 & \\
\hline & Antidepressivo & 3 & 30 & \\
\hline & Ansiolítico & 1 & 10 & \\
\hline & Diurético & 3 & 30 & \\
\hline & Dislipidemia & 3 & 30 & \\
\hline & Anticoagulante & 4 & 40 & \\
\hline & Anti-hipertensivo & 8 & 80 & \\
\hline Tabagismo & Sim & 4 & 40 & \\
\hline Etilismo & Sim & 3 & 30 & \\
\hline \multirow[t]{2}{*}{ Hemiparesia } & Esquerda & 5 & 50 & \\
\hline & Direita & 5 & 50 & \\
\hline \multirow[t]{4}{*}{ Locomoção } & Cadeira de rodas & 3 & 30 & \\
\hline & Andador & 1 & 10 & \\
\hline & Muletas & 1 & 10 & \\
\hline & Independente & 5 & 50 & \\
\hline \multirow[t]{2}{*}{ Tipo de AVC } & Isquêmico & 10 & 100 & \\
\hline & Hemorrágico & 0 & 0 & \\
\hline \multirow[t]{11}{*}{ Região do AVC } & Coroa radiata esquerda & 2 & 20 & \\
\hline & Centro sinovial esquerdo & 1 & 10 & \\
\hline & Lobo occipital esquerdo & 1 & 10 & \\
\hline & Hemisfério cerebelar direito & 2 & 20 & \\
\hline & Ponte & 1 & 10 & \\
\hline & Núcleos da base direito & 1 & 10 & \\
\hline & $\begin{array}{l}\text { Intraparenquimatosa em artéria cerebral média direita } \\
\text { Cápsula interna esquerda }\end{array}$ & 1 & 10 & \\
\hline & Núcleo caudado & 1 & 10 & \\
\hline & Tálamo & 2 & 20 & \\
\hline & Frontotemporal & 1 & 10 & \\
\hline & & 2 & 20 & \\
\hline
\end{tabular}

Tempo AVC (em dias)

$63,6 \pm 55,45$

Tempo de internação hospitalar (em dias)

$5,1 \pm 2,91$

\begin{tabular}{llr}
\hline AVC prévio & Sim & 3 \\
\hline
\end{tabular}

MEEM (pontuação)

$25,4 \pm 2,01$

DP: Desvio Padrão. n: Tamanho da amostra. \%: Porcentagem. HAS: Hipertensão Arterial Sistêmica. AVC: Acidente Vascular Cerebral. MEEM: Mini Exame do Estado Mental. 
Tabela 2. Caracterização sociodemográfica dos familiares.

\begin{tabular}{|c|c|c|c|c|}
\hline Variáveis & & $\mathbf{n}$ & $\%$ & Média e DP \\
\hline Idade & & & & $42,6 \pm 11,92$ \\
\hline Sexo & $\begin{array}{l}\text { Feminino } \\
\text { Masculino }\end{array}$ & $\begin{array}{l}8 \\
2\end{array}$ & $\begin{array}{l}80 \\
20\end{array}$ & \\
\hline Raça/cor & $\begin{array}{l}\text { Branca } \\
\text { Parda } \\
\text { Preta } \\
\text { Outra }\end{array}$ & $\begin{array}{l}2 \\
1 \\
3 \\
4\end{array}$ & $\begin{array}{l}20 \\
10 \\
30 \\
40\end{array}$ & \\
\hline Estado civil & $\begin{array}{l}\text { Solteiro } \\
\text { Casado } \\
\text { Outro }\end{array}$ & $\begin{array}{l}2 \\
6 \\
2\end{array}$ & $\begin{array}{l}20 \\
60 \\
20\end{array}$ & \\
\hline Anos de estudo & $\begin{array}{l}0 \text { a } 4 \\
5 \text { a } 8 \\
\text { Mais de } 9\end{array}$ & $\begin{array}{l}1 \\
3 \\
6\end{array}$ & $\begin{array}{l}10 \\
30 \\
60\end{array}$ & \\
\hline Trabalha & Sim & 6 & 60 & \\
\hline Parou de trabalhar para cuidar do paciente & Sim & 1 & 10 & \\
\hline MEEM (Pontuação) & & & & $27,4 \pm 2,24$ \\
\hline
\end{tabular}

Na Tabela 3 é apresentada a pontuação geral da PCRS dos 10 pacientes e familiares. Observou-se que alguns pacientes obtiveram diferença na pontuação na PCRS quando comparado ao seu familiar, com variação de 0 a 9 pontos. No geral, obtiveram pouca diferença na pontuação

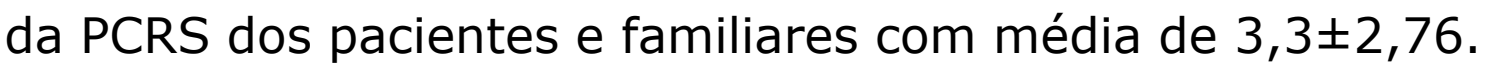

Tabela 3. Dados gerais da PCRS dos pacientes e familiares

\begin{tabular}{lll}
\hline Variáveis & Média e DP & Mínimo Máximo \\
\hline Pontuação PCRS pacientes & $75,7 \pm 6,03$ & \\
\hline Pontuação PCRS familiares & $74,2 \pm 6,82$ & \\
\hline Diferença pontuação PCRS pacientes e familiares & $3,3 \pm 2,76$ & 0 \\
\hline & & 0 \\
\hline
\end{tabular}


$\mathrm{Na}$ Tabela 4 evidencia-se os dados sociodemográficos

por caso dos pacientes e PCRS dos pacientes e respectivos familiares. Observa-se que os pacientes e seus respectivos familiares apresentaram discrepância na pontuação da PCRS que variou de 0 a 9 pontos.

Tabela 4. Dados sociodemográficos por caso dos pacientes e PCRS dos pacientes e respectivos familiares.

\begin{tabular}{|c|c|c|c|c|c|c|c|c|c|c|c|c|}
\hline $\mathbf{P}$ & I & Sexo & AE & Tab & Etil & Hemi & $\mathbf{L}$ & MEEM & PCRS-P & PCRS-F & $\begin{array}{l}\text { D PCRS-P } \\
\text { e PCRS-F }\end{array}$ & Visão P \\
\hline 1 & 55 & $\mathrm{~F}$ & 0 a 4 & $\mathrm{~N}$ & $\mathrm{~N}$ & $D$ & $\mathrm{C}$ & 22 & 75 & 81 & 6 & $\mathrm{Neg}$ \\
\hline 2 & 49 & $\mathrm{~F}$ & 0 a 4 & S & $\mathrm{N}$ & D & Ind & 27 & 81 & 76 & 5 & Pos \\
\hline 3 & 74 & $\mathrm{~F}$ & 5 a 8 & S & $\mathrm{N}$ & D & C & 26 & 80 & 71 & 9 & Pos \\
\hline 4 & 56 & $\mathrm{~F}$ & 0 a 4 & $\mathrm{~S}$ & $\mathrm{~N}$ & D & Ind & 23 & 83 & 81 & 2 & Peq D \\
\hline 5 & 60 & $\mathrm{~F}$ & 0 a 4 & $\mathrm{~N}$ & $\mathrm{~N}$ & E & C & 23 & 68 & 68 & 0 & $\mathrm{Ig}$ \\
\hline 6 & 29 & $M$ & 5 a 8 & $\mathrm{~N}$ & $\mathrm{~N}$ & $E$ & Ind & 26 & 72 & 71 & 1 & Peq D \\
\hline 7 & 71 & $\mathrm{~F}$ & 5 a 8 & $\mathrm{~N}$ & $\mathrm{~N}$ & $E$ & A & 26 & 64 & 62 & 2 & Peq D \\
\hline 8 & 78 & $M$ & 5 a 8 & $S$ & $S$ & $E$ & Ind & 26 & 73 & 68 & 5 & Pos \\
\hline 9 & 50 & $M$ & 5 a 8 & $\mathrm{~N}$ & $\mathrm{~S}$ & D & $M$ & 26 & 80 & 83 & 3 & $\mathrm{Neg}$ \\
\hline 10 & 32 & $M$ & $\begin{array}{l}\text { Mais } \\
\text { de } 9\end{array}$ & $\mathrm{~N}$ & S & $E$ & Ind & 29 & 81 & 81 & 0 & Ig \\
\hline
\end{tabular}

P: Paciente. I: idade. F: Feminino. M: Masculino. AE: Anos de Estudo. Tab.: Tabagismo. S: Sim. N: Não. Etil: Etilismo. Hemi: Hemiparesia. D: Direita. E: Esquerda. L: Locomoção. C: Cadeira de rodas. A: Andador. M: Muleta. Ind: Independente. MEEM: Mini Exame do Estado Mental. PCRS-P: The Patient Competency Rating Scale Paciente. PCRS-F: The Patient Competency Rating Scale Familiar. D PCRS-P e PCRS-F: Discrepância PCRS paciente e familiar. Visão P: Visão paciente sobre si. Ig: Igual. Pos: Positiva. Neg: Negativa. Peq D: Pequena discrepância. 
Na Tabela 5 são apresentados os dados sociodemográficos dos cinco pacientes do Grupo Possível Anosognosia (GPA) e cinco pacientes do Grupo Não Possível Anosognosia (GNPA), ao assumir que pontuações discrepantes de igual ou maior a 3 pontos entre os pacientes e familiares, podem ser sugestivos de anosognosia. Visto que não é possível comprovar estatisticamente por não existir escore de classificação na PCRS.

\section{DISCUSSÃO}

Em um estudo de coorte prospectivo com 58 pacientes, no qual foi avaliada a anosognosia pós AVC no hemisfério direito, foi observado que a anosognosia foi mais frequente na fase hiperaguda aos três dias com $32 \%$, na fase subaguda aos sete dias com $18 \%$ e na fase crônica aos seis meses com $5 \%{ }^{13}$. Portanto, optou-se por realizar o estudo na fase aguda e subaguda do AVC devido a maior ocorrência de anosognosia, de acordo com a literatura.

Um estudo com 302 pacientes analisou as sequelas psicológicas, principalmente qualidade de vida e o humor após um AVC e observou que $22 \%$ dos pacientes apresentaram sintomas depressivos e aproximadamente $32 \%$ relataram ansiedade moderada ${ }^{10}$. Alguns pacientes deste estudo apresentaram doenças psicológicas após o AVC, pelo fato de que $30 \%$ faziam o uso de medicação antidepressiva e $10 \%$ faziam uso de medicação ansiolítica. 
Tabela 5. Caracterização sociodemográfica dos pacientes no Grupo Possível Anosognosia (GPA) e Grupo Não Possível Anosognosia (GNPA).

\begin{tabular}{|c|c|c|c|c|}
\hline \multicolumn{2}{|l|}{ Variáveis } & $\begin{array}{l}\text { GPA } \\
(n=5)\end{array}$ & $\begin{array}{l}\text { GNPA } \\
(n=5)\end{array}$ & $\begin{array}{c}\text { Total } \\
(n=10)\end{array}$ \\
\hline \multicolumn{2}{|c|}{ Idade (média e DP) } & $61,2 \pm 12,31$ & $49,6 \pm 16,38$ & \\
\hline Sexo & $\begin{array}{l}\text { Feminino } \\
\text { Masculino }\end{array}$ & $\begin{array}{l}3(60 \%) \\
2(40 \%)\end{array}$ & $\begin{array}{l}3(60 \%) \\
2(40 \%)\end{array}$ & \\
\hline Raça/cor & $\begin{array}{l}\text { Branca } \\
\text { Parda } \\
\text { Preta } \\
\text { Outra }\end{array}$ & $\begin{array}{l}1(20 \%) \\
1(20 \%) \\
1(20 \%) \\
2(40 \%)\end{array}$ & $\begin{array}{l}1(20 \%) \\
1(20 \%) \\
0(0 \%) \\
3(60 \%)\end{array}$ & \\
\hline Estado civil & $\begin{array}{l}\text { Solteiro } \\
\text { Casado } \\
\text { Separado } \\
\text { Outro }\end{array}$ & $\begin{array}{l}1(20 \%) \\
2(40 \%) \\
1(20 \%) \\
1(20 \%)\end{array}$ & $\begin{array}{l}2(40 \%) \\
3(60 \%) \\
0(0 \%) \\
0(0 \%)\end{array}$ & \\
\hline Anos de estudo & $\begin{array}{l}0 \text { a } 4 \\
5 \text { a } 8 \\
\text { Mais de } 9\end{array}$ & $\begin{array}{l}2(40 \%) \\
3(60 \%) \\
0(0 \%)\end{array}$ & $\begin{array}{l}2(40 \%) \\
2(40 \%) \\
1(20 \%)\end{array}$ & \\
\hline Comorbidades & $\begin{array}{l}\text { HAS } \\
\text { Fibrilação Atrial } \\
\text { Doença Arterial Coronariana } \\
\text { Diabetes } \\
\text { Depressão } \\
\text { Obesidade } \\
\text { Arritmia Cardíaca } \\
\text { Flutter Atrial } \\
\text { ICC }\end{array}$ & $\begin{array}{l}5(100 \%) \\
1(20 \%) \\
0(0 \%) \\
0(0 \%) \\
1(20 \%) \\
0(0 \%) \\
1(20 \%) \\
0(0 \%) \\
0(0 \%)\end{array}$ & $\begin{array}{l}2(40 \%) \\
2(40 \%) \\
1(20 \%) \\
2(20 \%) \\
0(0 \%) \\
1(20 \%) \\
0(0 \%) \\
1(20 \%) \\
1(20 \%)\end{array}$ & \\
\hline Medicamentos & $\begin{array}{l}\text { Antidiabético } \\
\text { Antidepressivo } \\
\text { Ansiolítico } \\
\text { Diurético } \\
\text { Dislipidemia } \\
\text { Anticoagulante } \\
\text { Anti-hipertensivo }\end{array}$ & $\begin{array}{l}0(0 \%) \\
2(40 \%) \\
1(20 \%) \\
1(20 \%) \\
2(40 \%) \\
2(40 \%) \\
5(100 \%)\end{array}$ & $\begin{array}{l}2(40 \%) \\
1(20 \%) \\
0(0 \%) \\
2(40 \%) \\
1(20 \%) \\
2(40 \%) \\
3(60 \%)\end{array}$ & \\
\hline Tabagismo & $\begin{array}{l}\text { Não } \\
\text { Sim }\end{array}$ & $\begin{array}{l}2(40 \%) \\
3(60 \%)\end{array}$ & $\begin{array}{l}4(80 \%) \\
1(20 \%)\end{array}$ & \\
\hline Etilismo & $\begin{array}{l}\text { Não } \\
\text { Sim }\end{array}$ & $\begin{array}{l}3(60 \%) \\
2(40 \%)\end{array}$ & $\begin{array}{l}4(80 \%) \\
1(20 \%)\end{array}$ & \\
\hline Hemiparesia & $\begin{array}{l}\text { Esquerda } \\
\text { Direita }\end{array}$ & $\begin{array}{l}1(20 \%) \\
4(80 \%)\end{array}$ & $\begin{array}{l}4(80 \%) \\
1(20 \%)\end{array}$ & \\
\hline Locomoção & $\begin{array}{l}\text { Cadeira de rodas } \\
\text { Andador } \\
\text { Muletas } \\
\text { Independente }\end{array}$ & $\begin{array}{l}2(40 \%) \\
0(0 \%) \\
1(20 \%) \\
2(40 \%)\end{array}$ & $\begin{array}{l}1(20 \%) \\
1(20 \%) \\
0(0 \%) \\
3(60 \%)\end{array}$ & \\
\hline \multicolumn{2}{|c|}{ Tempo AVC (em dias)(média e DP) } & $79,2 \pm 52,9$ & $25,8 \pm 53,43$ & \\
\hline \multicolumn{2}{|c|}{ Dias de internação hospitalar (média e DP) } & $5,8 \pm 3,54$ & $4,4 \pm 1,85$ & \\
\hline AVC prévio & $\begin{array}{l}\text { Não } \\
\text { Sim }\end{array}$ & $\begin{array}{l}3(60 \%) \\
2(40 \%)\end{array}$ & $\begin{array}{l}4(80 \%) \\
1(20 \%)\end{array}$ & \\
\hline MEEM (pontuaçą & ia e DP) & $25,4 \pm 1,74$ & $25,4 \pm 2,24$ & \\
\hline
\end{tabular}

DP: Desvio Padrão. n: Tamanho da amostra. \%: Porcentagem. HAS: Hipertensão Arterial Sistêmica. AVC: Acidente Vascular Cerebral. ICC: Insuficiência Cardíaca Congestiva. MEEM: Mini Exame do Estado Mental. GPA: Grupo Possível Anosognosia. GNPA: Grupo Não Possível Anosognosia. 
A autoconsciência pode ser definida como um processo constante de monitoramento interno e externo dos eventos. Quanto maior o nível de autoconsciência do indivíduo, maior a possibilidade de apresentar relação com o bem-estar psicológico. Contrariamente, na redução da autoconsciência pode existir o sofrimento psicológico ${ }^{11}$.

É importante avaliar a autoconsciência, porque ela afeta a capacidade do paciente abordar os processos emocionais, cognitivos, motores e a funcionalidade. Quando a autoconsciência está prejudicada existem dificuldades no cumprimento da reabilitação neuropsicológica e consequentemente na integração com a comunidade ${ }^{16}$. Acredita-se que os pacientes que se enxergam com mais facilidade para realizar as atividades como realmente são, por acreditarem que não apresentem muita dificuldade, podem não aderir a reabilitação ou tratamento clínico, e com isso, não apresentarem bons resultados na restauração da funcionalidade. No entanto, os pacientes que não possuem anosognosia têm mais chances de aderir à reabilitação e apresentam consciência da importância e consequentemente melhores resultados na recuperação.

Um estudo avaliou a autoconsciência que os indivíduos hospitalizados após o AVC possuem sobre seus déficits e sua relação com a ansiedade e depressão, por meio de escalas e uma entrevista e, foi observada correlação entre a autoconsciência e sintomas depressivos ${ }^{9}$.

Ao analisar a discrepância da pontuação PCRS entre paciente e familiar, observou-se que dois (20\%) não 
apresentam diferença (0 pontos), ou seja, paciente e familiar têm a mesma noção das dificuldades que o paciente apresenta, e o paciente não apresenta anosognosia. Já, três (30\%) dos pacientes apresentaram pequena variação na pontuação da PCRS comparada aos familiares, as pontuações variaram de $1,2,2$ respectivamente, e considerando não haver um escore que permita a comparação, considerou-se que estes pacientes não apresentam anosognosia, pela baixa variação nas respostas entre familiar e paciente.

Contudo, dois (20\%) dos pacientes tiveram pontuação mais baixa na PCRS que os familiares variando de 3 a 6 , e pela variação entre as respostas possuem anosognosia, e acreditam ter mais dificuldade em realizar as atividades questionadas pela PCRS. No entanto, três (30\%) dos pacientes obtiveram pontuação mais alta na PCRS que os familiares, as pontuações variaram 5, 5 e 9, e por isso considerou-se que essa discrepância indica a anosognosia, e acreditam ter mais facilidade em realizar as atividades questionadas pela PCRS. Portanto, considerou-se que cinco (50\%) dos pacientes apresentam anosognosia. Não é possível comprovar estatisticamente por não existir escore de classificação na PCRS, contudo sugere-se que pontuações discrepantes de igual ou maior a 3 pontos entre os pacientes e familiares, podem ser sugestivos de anosognosia.

Ao analisar a discrepância da pontuação dos pacientes e familiares na PCRS por item, o item com menor variação dos resultados foi o item de número 13 , com zero pontos. 0 
item 13 aborda "Quanta dificuldade você tem em perceber que algo que disse ou fez chateou outra pessoa?". Portanto, observou-se que os pacientes possuem mais consciência nas atividades de automonitoramento. Já que o item 13 da PCRS aborda o componente cognitivo auto-monitoramento ${ }^{15}$.

Os itens com maior variação dos resultados da pontuação da PCRS dos pacientes e familiares foram os de números 16 e 17, ambos com nove pontos. 0 item 16 aborda "Quanta dificuldade você tem em cumprir de maneira efetiva as suas responsabilidades diárias?". O item 17 aborda "Quanta dificuldade você tem em se manter concentrado em uma atividade ou tarefa? Por exemplo, quando quer ver um capítulo de novela até o final ou assistir um programa de televisão até o final. Portanto, observou-se que os pacientes possuem menos consciência nas atividades de planejamento e atenção sustentada. Visto que o item 16 da PCRS aborda o componente cognitivo planejamento e o item 17 aborda a atenção sustentada ${ }^{15}$.

As duas pacientes ( 1 e 3 ) com maior diferença na PCRS eram do sexo feminino, tinham média de idade $64,5 \pm 9,5$, tinham baixa escolaridade ( 0 a 8 anos de estudo), HAS, não tinham AVC prévio, hemiparesia à direita, utilizavam cadeira de rodas para locomoção e a discrepância na PCRS foi de 6 e 9 pontos, respectivamente. Com isso, apresentam média de idade mais elevada. As pacientes apresentam HAS, o que pode ter favorecido a ocorrência do AVC pela baixa escolaridade ( 0 a 8 anos de estudo), que compromete 0 conhecimento sobre medidas de prevenção de doenças, bem 
como reconhecimento de sinais e sintomas ${ }^{17}$. E apresentam comprometimento no nível motor pela hemiparesia à direita e uso de cadeira de rodas para locomoção.

Entre os cinco pacientes do GPA, possuem média de $61,2 \pm 12,31$ anos, três (60\%) fazem uso de ansiolítico ou antidepressivo, quatro (80\%) apresentam hemiparesia à direita e dois (40\%) possuem locomoção independente. Em relação aos cinco pacientes do GNPA, possuem média de

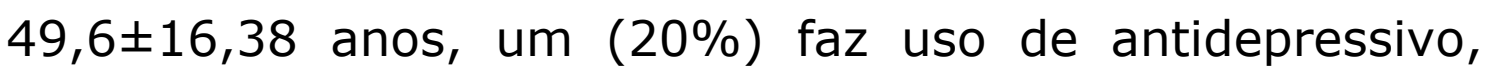
quatro (80\%) apresentam hemiparesia à esquerda e três (60\%) possuem locomoção independente.

Quanto ao tabagismo, no GPA três (60\%) dos pacientes são tabagistas e no GNPA um (20\%) é tabagista. Em relação ao etilismo, dois (40\%) do GPA são etilistas e um (20\%) do GNPA é etilista.

Neste estudo, ao analisar a região do cérebro acometida pelo AVC, elas só foram iguais em duas pessoas que, no entanto, na pontuação final do PCRS se apresentaram em grupos diferentes (GPA e GNPA). Portanto, neste estudo não se pode afirmar que exista relação com a presença no GPA ou GNPA e a região acometida.

Um estudo investigou se os pacientes pós AVC estavam conscientes das suas habilidades para o ato de dirigir. Contou com 30 pacientes pós AVC com três meses de lesão e um grupo controle saudável. Os pacientes foram avaliados num simulador de direção e foram questionados sobre como se percebiam como motoristas quando comparado a outra pessoa de mesma idade, sem AVC, a pergunta foi realizada 
antes e após o teste no simulador. Antes e depois de passar pelo teste do simulador, 53\% dos pacientes com AVC acreditavam ser melhores motoristas que o controle, houve uma superestimação dos resultados pelos pacientes em pelo menos 10 pontos, quando comparado com controle. No grupo controle apenas $16 \%$ acreditavam que eram melhores que a população em geral após o teste. Conclui-se que a maioria das pessoas com AVC tem reduzida consciência dos seus déficits de condução, o que pode causar riscos à saúde, sendo mais comum reconhecer os déficits físicos do que os $\operatorname{cognitivos}^{12}$. Esses achados estão de acordo com este estudo, em que se considerou que 30\% dos pacientes apresentam anosognosia após AVC, uma vez que acreditam apresentar mais facilidade em realizar algumas atividades de vida diária.

Um estudo investigou a anosognosia pós AVC em 11 pacientes de um grupo de reabilitação, com média de idade de $62,73 \pm 8$. Foi aplicada a PCRS na versão brasileira, houve discrepância da PCRS entre pacientes e familiares de 9,45士6 pontos. Os pacientes apresentam indicativos de alteração de percepção em relação às dificuldades. Os pacientes apresentam uma visão positiva em $73 \%$, e negativa em $27 \%$ em relação à sua saúde. Contudo, não afirmam estatisticamente, devido a PCRS não apresentar escore de classificação ${ }^{18}$. Esses dados se assemelham ao presente estudo, em que se considerou que alguns pacientes apresentam anosognosia e tendem a se enxergar de maneira mais positiva. 
A anosognosia e a heminegligência estão associadas a disfunção do hemisfério direito e geralmente ao lobo parietal. A anosognosia é uma síndrome comportamental bastante frequente após o AVC no hemisfério direito ${ }^{19}$. Neste estudo, o paciente com maior diferença na PCRS com o familiar, que foi de 9 pontos, apresentava lesão no hemisfério esquerdo, e consequentemente hemiparesia à direita, contraditoriamente ao apontado na literatura. No GPA, quatro $(80 \%)$ dos pacientes possuíam hemiparesia à direita e lesão no hemisfério esquerdo, o que não apresenta relação com a literatura. Entretanto, neste estudo não foi possível investigar a relação da anosognosia com o hemisfério cerebral acometido, tanto pela amostra ser reduzida, quanto e principalmente devido a PCRS não existir escore de classificação, fato que impede a comprovação da anosognosia, mas sugere que ela esteja presente pela discrepância paciente/familiar. Neste sentido, sugere-se que estudos mais amplos sejam realizados para relacionar a presença da anosognosia com os hemisférios cerebrais.

Além disso, um estudo mostrou que em indivíduos com anosognosia é observada a dificuldade no engajamento no processo da reabilitação e no resultado obtido ${ }^{20}$. É importante existir a autoconsciência dos déficits após o AVC, porque permite a segurança física do indivíduo, evita a exposição de riscos, estimulando a adesão ao tratamento clínico e à reabilitação ${ }^{21}$.

Sugere-se que estudos com amostra mais abrangente sejam realizados para que assim possam ser estabelecidas 
relações estatísticas. Essa, portanto, é apontada como a maior fragilidade deste estudo. Da mesma maneira, sugerese que estudos sobre anosognosia em pacientes com AVC, adotem como critério de inclusão dos participantes, a avaliação da cognição por meio do MEEM, como um instrumento amplamente utilizado e de alta confiabilidade, garantindo que os participantes estejam ofertando respostas concretas e conscientes sobre a sua saúde e dos seus familiares.

\section{CONCLUSÕES}

O estudo pode concluir que $20 \%$ dos pacientes com AVC na fase aguda e subaguda não possuem anosognosia por não apresentarem diferença na PCRS entre paciente e familiar, assim como os outros $30 \%$ pela mínima diferença nos resultados. Entre os fatores relacionados a autopercepção positiva do seu estado geral de saúde se destacou como elemento considerável para a presença de anosognosia.

De acordo com o presente estudo, a anosognosia pode apresentar relação com a idade mais avançada, uso de antidepressivo ou ansiolítico, hemiparesia à direita, maior dependência para locomoção e influência do tabagismo e etilismo.

\section{AGRADECIMENTOS}

Agradecemos ao Hospital Casa de Saúde de Santa Maria, e à Prefeitura Municipal de Santa Maria em que a UBS 
Floriano da Rocha e ESF da Vila Kennedy estão vinculadas, pela colaboração com o estudo.

\section{REFERÊNCIAS}

1.Santos LB, Waters C. Perfil epidemiológico dos pacientes acometidos por acidente vascular cerebral: revisão integrativa. Braz J Develop 2020;6:2749-75. http://dx.doi.org/10.34117/bjdv6n1-198

2.Pang MY, Eng JJ, Dawson AS, Gylfadóttir S. The use of aerobic exercise training in improving aerobic capacity in individuals with stroke: a meta-analysis. Clin Rehabil 2006;20:97-111. http://dx.doi.org/10.1191/0269215506cr926oa

3.Damata SRR, Formiga LMF, Araújo AKS, Oliveira EAR, Oliveira AKS, Formuga RCF. Perfil epidemiológico dos idosos acometidos por acidente vascular cerebral. Rev Interd 2016;9:107-7. https://revistainterdisciplinar.uninovafapi.edu.br/index.php/revinter/a rticle/view/751/pdf 283

4.Lima SM, Maldonade I. Avaliação da linguagem de pacientes no leito hospitalar depois do acidente vascular cerebral. Distúrb Comun 2016;28:673-85.

https://revistas.pucsp.br/index.php/dic/article/view/27555/21991

5.Prigatano GP. The Study of Anosognosia. New York: Oxford University Press, 2010. https://books.google.com.br/books?id=d4STONboMQC\&printsec $=$ frontcover\&hl=pt-

BR\&source $=$ gbs ge summary $r \& c a d=0 \# v=$ onepage $\& q \& f=$ false

6.Vossel S, Weiss PH, Eschenbeck P, Fink GR. Anosognosia, neglect, extinction and lesion site predict impairment of daily living after right hemispheric stroke. Cortex 2013;49:1782-9.

http://dx.doi.org/10.1016/j.cortex.2012.12.011

7.Starkstein SE, Jorge RE, Robinson R. The Frequencies, Clinical Correlates, and Mechanism of Anosognosia After Stroke. Can J Psychiatric 2010;55:355-61. http://dx.doi.org/10.1016/j.cortex. 2014.10 .017

8.Cocchini G, Beschin N, Cameron A, Fotopoulou A, Sala SD. Anosognosia for motor impairment following left brain damage. Neuropsychology 2009;23:223-30.

http://dx.doi.org/10.1037/a0014266

9. Vieira AA, Pereira APA. Anosognosia e sintomas de ansiedade e depressão em pessoas após Acidente Vascular Cerebral. (Dissertação). Curitiba: Universidade Federal do Paraná; 2018. https://acervodigital. ufpr.br/bitstream/handle/1884/58649/R\%20$\% 20 \mathrm{\%} 20-$

$\%$ 20ANDRESSA \%20ALINE\%20VIEIRA.pdf? sequence $=1$ \&isAllowed $=y$ 10.Mellon L, Willians D, Brewer P, Hall P, Horgan F, Dolan E, et al. Psychological symptoms and quality of life post-stroke. A profile of Irish stroke survivors from the ASPIRE-S cohort. J Psychosom Res 2016;85:74-84. http://dx.doi.org/10.1016/j.jpsychores.2016.03.182 
11.DaSilveira A, DeSouza ML, Gomes, WB. Self-consciousness concept and assessment in self-report measures. Front Psychol 2015;6:1-11. http://dx.doi.org/10.3389/fpsyg.2015.00930

12.McKay C, Rapport LJ, Bryer RC, Casey J. Self-evaluation of driving simulator performance after stroke. Top Stroke Rehabil 2011;18:54961. http://dx.doi.org/10.1310/tsr1805-549

13.Vocat R, Staub F, Stroppini T, Vuilleumier P. Anosognosia for hemiplegia: a clinical-anatomical prospective study. Brain 2010;133:3578-97. http://dx.doi.org/10.1093/brain/awq297

14.Crum RM, Anthony JC, Bassett SS, Folstein MF. Population-Based Norms for the Mini-Mental State Examination by Age and Educational Level. Jama 1993;269:2386-91.

http://dx.doi.org/10.1001/jama.1993.03500180078038

15.Zimmermann N, Pereira APA, Fonseca RP. Brazilian Portuguese version of the Patient Competency Rating Scale (PCRS-R-BR): semantic adaptation and validity. Trends Psychiatr Psychother. 2014;36:40-51. http://dx.doi.org/10.1590/2237-6089-2013-0021

16. Bivona U, Ciurli P, Barba C, Onder G, Azicnuda E, Silvestro D, et al. Executive function and metacognitive self-awareness after severe traumatic brain injury. J Int Neuropsychol Soc 2008;14:862-8. http://dx.doi.org/10.1017/S1355617708081125

17.Silva IFG, Neves CFS, Vilela ACG, Bastos LMD, Henriques MILS. Viver cuidar após o acidente vascular cerebral. Rev Enf Ref 2016;4:103-11. http://dx.doi.org/10.12707/RIV15047

18. Keller GR, Dias GM, Prado ALC, Silva HT, Pacheco CB. Investigação da Anosognosia Pós Acidente Vascular Cerebral em Participantes de Um Grupo de Reabilitação. Rev Saúde (Sta. Maria) 2019;45:1-13. http://dx.doi.org/10.5902/2236583438177

19.Palmerini $\mathrm{F}$, Bogousslavsky J. Right hemisphere syndromes. In: Paciaroni M, Agnelli G, Caso V, Bogousslavsky J (eds). Manifestations of stroke. Front Neurol Neurosci 2012;30:61-4. http://dx.doi.org/10.1159/000333411

20.Bevilacqua F, Bisello A, Chinellato A, Pilosio C, Meneghello F. Lack of awareness in brain injured patients: the importance of a multidisciplinary assessment in the functional autonomy recovery. Brain Injury 2008;44:1-3. https://www.researchgate. net/publication/237334723 Lack of awar eness in brain injiured patients the importance of a multidisciplin ary assessment in the functional autonomy recovery

21.Barrett AM, Galletta EE, Zhang J, Masmela JR, Adler US. Stroke Survivors Over-estimate Their Medication Self-Administration (MSA) Ability, Predicying Memory Loss. Brain Injury 2014;28:1328-33. http://dx.doi.org/10.3109/02699052.2014.915984 